\title{
Determination of Spectral Power Distribution and Spectral Mismatch Factor for Some Commercial Compact Fluorescent Lamps
}

\author{
Manal A. Haridy (Phd) ${ }^{1,2^{*}}$, Hanan R. Alenzi(Msc) ${ }^{2}$, Layla R. Altamimi(Msc) ${ }^{2}$, \\ Wasyuf M. Alenzy(Msc) ${ }^{2}$ \\ ${ }^{1}$ Photometry and Radiometry Division, National Institute of Standards (NIS), Giza, Egypt Physics Department, \\ 2 College of Science, University of Hail (UOH), Hail, Kingdom of Saudi Arabia (KSA). \\ *Corresponding Author: manal_haridi@yahoo.com
}

\begin{abstract}
The present research aimed to investigate some lighting characteristics of six commercial compact fluorescent lamps (CFLs). The lighting characteristics such as spectral power distributions (SPDs), and spectral mismatch correction factor (SCF) to human response eye curve $V(\lambda)$ have important roles on visual health effects of the lamps. A set up based on National Institute of standards of Egypt (NIS) Spectroradiometer ocean optics HR 2000 with uncertainty $4.7 \%$ and the photometric bench has been used for measuring the spectral power distribution of the lamps to determine the spectral mismatch correction factor. Calculations of comparative analysis have been done for spectral mismatch correction factor (SCF) to human response curve V $(\lambda)$. The spectral power distributions (SPDs) diagrams for the commercial compact lamps (CFLs) showed typical CFLs response, with a few narrow spectral peaks. The histogram for comparison of spectral mismatch factor shows the spectral mismatch factor for each CFL lamp and the degree of perfectly spectral matching with human response eye curve $\mathrm{V}(\lambda)$. Data analysis was performed and uncertainty model includes all parameters accompanied with the measurements are calculated.

Keywords: Spectral Power Distribution (SPD), Spectral mismatch correction factor (SCF), Compact Fluorescent Lamps (CFLs), Human response curve V $(\lambda)$, Uncertainty,
\end{abstract}

\section{INTRODUCTION}

Lighting energy can be saved in many ways, including improving the efficiency of the light source, improving the efficiency of the specific component of lighting system, typically the ballast and, improving the efficiency of the luminaries [1,2]. Replacing technology can occur at a very low cost and provide immediate results [3]. Replacing incandescent lamps with compact fluorescent lamps (CFLs) which have played an important role in both industrial and domestic lighting is one of the most obvious and easiest methods to achieve energy efficiency [4]. As a best alternative for light bulb, compact fluorescent lamps (CFLs) bulbs are an excellent option. Compact fluorescent lamps (CFLs) are a miniature version of the common fluorescent light, glowing phosphor gas by using an electric current. Earlier the compact fluorescent lamps (CFLs) used magnetic ballasts which usually results into delay or flicker when turned on. Whereas, the most new compact fluorescent lamps (CFLs) use electronic ballasts. On comparing with incandescent bulbs, compact fluorescent lamps (CFLs) are approximately four times as efficient i.e. a 25 Watt CFL will have the same light output as a 100 Watt incandescent bulb. They also possesses high longevity i.e. 10 times longer, meaning that the life of a standard CFL is comparable to 10 incandescent bulbs. But, a CFL gives off light that looks just like a standard incandescent [5].

Lamp is usually compounded of two parts.

One is plastic cover with holes for pipe and bills. Tube is agglutinated to it. Second much bigger piece has slots for bills from the inner side. Inside is printed circuit board with components and wires from tube. From the upper side of PCB are wires to top of lamp, where are soldered or stamped to the contact. Usually you can carefully leverage with a small screwdriver sequently to round to the gap between both plastic pieces for releasing of glue. Next you must leverage more to the opening lamp. For closing of lamp you can only click both plastic pieces to himself. Look at photo of opened lamp. 


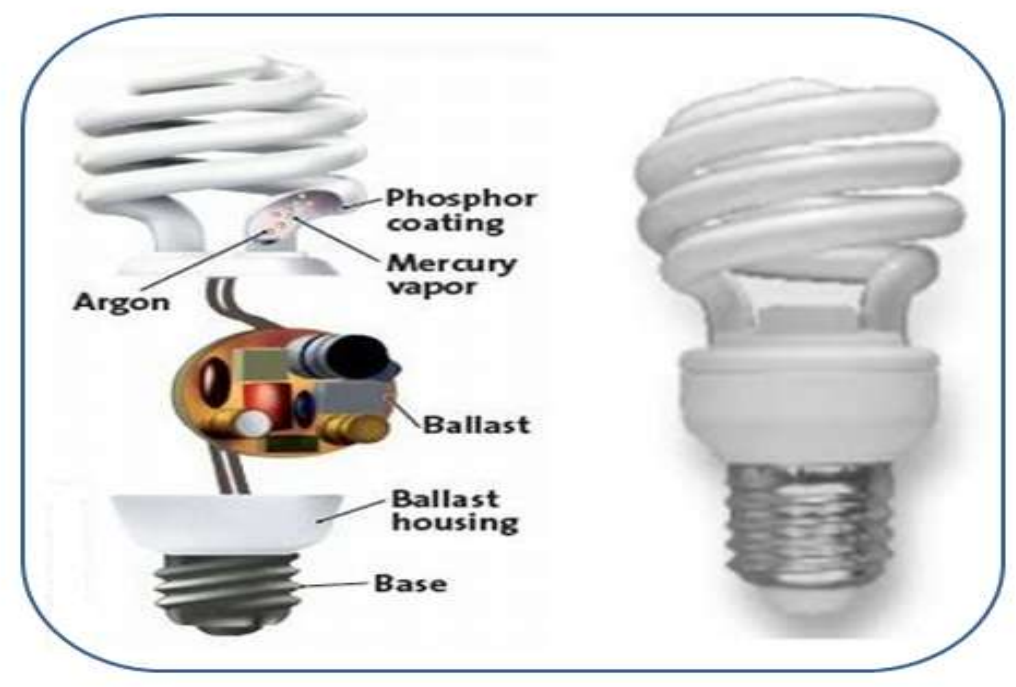

Figure 1. Schematic Diagram of Compact Fluorescent Lamp.

Spectral power distribution (SPD) is used for describing visible spectrum of lighting sources. This quantity shows the radiant power emitted by the source at each wavelength over the visible region (380 to $760 \mathrm{~nm}$ ). Lamp manufacturers present the SPD curves of specific light sources. The incandescent lamp frequently has high power in the longer wavelengths (above $650 \mathrm{~nm}$ ) of the visible spectrum and therefore, effectively renders red colors. The light emitting diode lamp has high power in the short wavelength of the visible spectrum (below $450 \mathrm{~nm}$ ) [6,7].

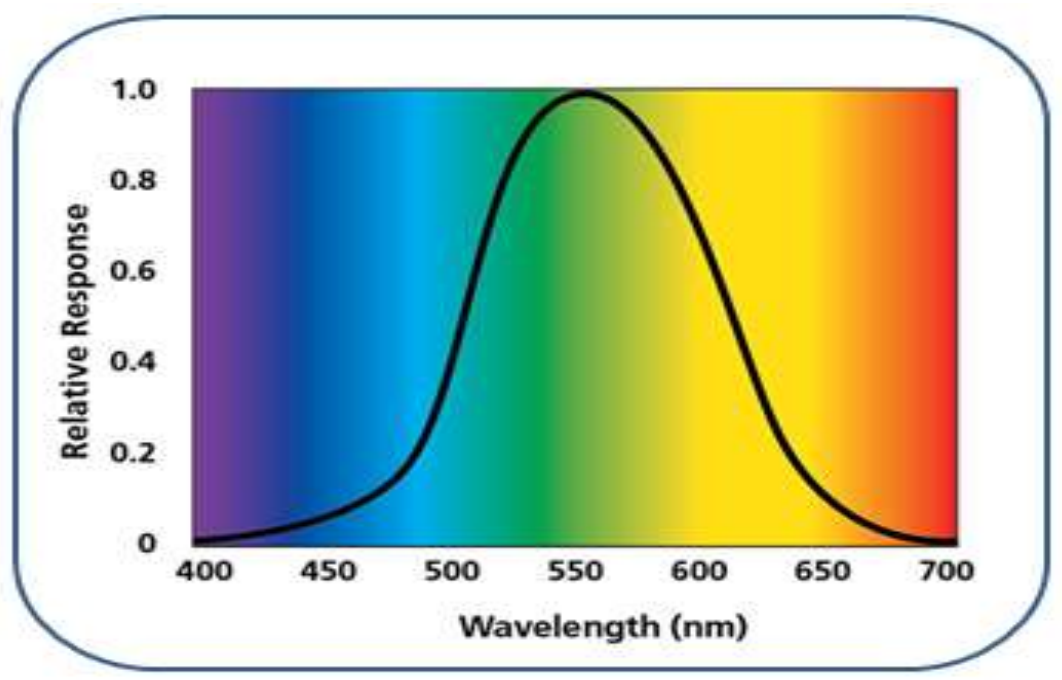

Figure. 2: CIE Photopic Response - Standard Observer Function for Photopic Vision

The spectral matching of photometers is important for photometric measurements. However, there is no general estimation for the spectral mismatch correction factor [8] for the measurement of CFLs. According to that, An error occurs when a photometer head measures a light source having the relative spectral power distribution (SPDs) different from the calibrated source. The spectral error can be taken into account through a spectral mismatch correction factor [8-10].

$$
S C F=\frac{\int_{360}^{830 \mathrm{~nm}} P_{e}^{T}(\lambda) \times V(\lambda) d \lambda \int_{\text {all -wavelength }}{ }_{s} P_{e}^{S}(\lambda) \times R(\lambda) d \lambda}{\int_{\text {all - wavelength }} P_{s} P_{e}^{T}(\lambda) \times R(\lambda) d \lambda \int_{360}^{830 \mathrm{~nm}} P_{e}^{S}(\lambda) \times V(\lambda) d \lambda}
$$

Where $P_{e}^{T}(\lambda):$ is the relative spectral output of the test source. 
$P_{e}^{S}(\lambda)$ : is the relative spectral output of the standard source.

$R(\lambda)$ : is the relative spectral responsivity of the photometer.

$V(\lambda):$ is the spectral luminous efficiency function.

\section{METHODS}

In the present research, a setup based on NIS Spectroradiometer ocean optics HR 2000 with uncertainty $4.7 \%[11]$ and the photometric bench for measuring the spectral power distribution of the six commercial compact fluorescent lamps (CFLs) as the following: NIS-UOH-CFL-1, NIS-UOH-CFL-2, NISUOH-CFL-3, NIS-UOH-CFL-4, NIS-UOH-CFL-5, NIS-UOH-CFL-6 and to determine the spectral mismatch correction factor, shown schematically in Figure (3). It measured directly using the photometric bench and spectroradiometer to evaluate the spectral power distribution. Light to be measured is guided into entrance port of spectroradiometer through an optical fiber and the spectrum is output through the USB port to a PC for a data acquisition. An optical fiber that guides light input from compact lamps (CFLs) allows a flexible measurements setup. The spectroradiometric measurement of light sources was performed based on CIE 63-1984 method recommended by International Electrotechnical Commission (IEC) [12]. The employed spectroradiometer is periodically calibrated., spectroradiometers are the most accurate for measuring spectral energy distribution of any light source. In this way, the studied lamps were separately installed at a distance of half a meter above the spectroradiometer and then after fifteen minutes, the interested data for each lamp was registered. Measurements were performed in a conditioned dark room and maintaining the temperature at $(25 \pm 2)^{\circ} \mathrm{C}$. The Set up of measuring the spectral power distribution lamps in Figure. 3.

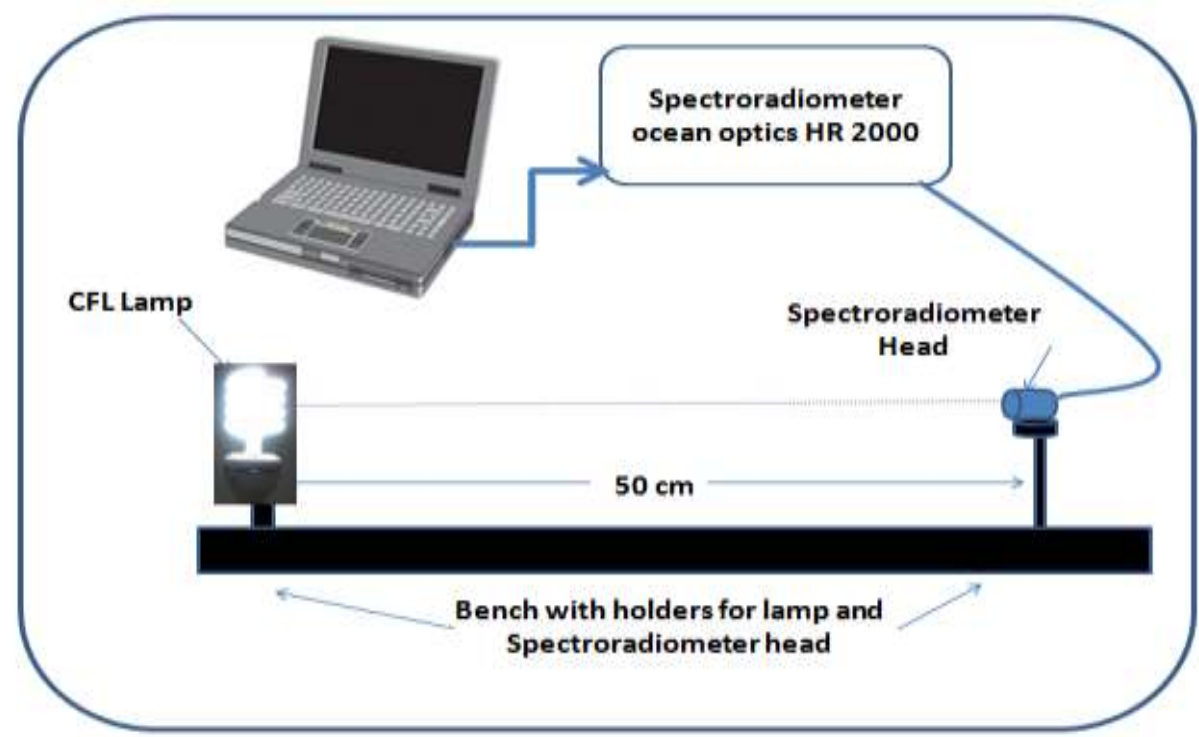

Figure. 3. Set up of measurements the spectral power distribution compact lamps (CFLs).

\section{RESULTS AND DISCUSSIONS}

A setup based on NIS spectral radiometers and photometric bench for measuring the spectral power distribution of the six commercial compact fluorescent lamps (CFLs) as the following: NIS-UOH-CFL-1, NISUOH-CFL-2, NIS-UOH-CFL-3, NIS-UOH-CFL-4, NIS-UOH-CFL-5, NIS-UOH-CFL-6.The relative spectral power distribution was normalized for each lamp so we can compare the lamps as shown in Figure (4).

Figure (4) shows spectral power distribution (SPDs) diagrams at each wavelength over the visible region of six of commercial compact fluorescent lamps as the following: NIS-UOH-CFL-1, NIS-UOH-CFL-2, NIS-UOH-CFL-3, NIS-UOH-CFL-4, NIS-UOH-CFL-5, NIS-UOH-CFL-6 compared to the spectral power distribution of the human response curve $\mathrm{V}(\lambda)$. It is used for describing visible spectrum of lighting sources. This quantity shows the radiant power emitted by the source at each wavelength over the visible region (400 to $700 \mathrm{~nm}$ ). The spectral power distribution (SPDs) diagrams showed typical compact fluorescent lamps (CFLs) response, with a few narrow spectral peaks. It is found that each group of lamps has its own characteristics and they emit their spectrum in the visible region with different spectral distributions for each lamp. 
Manal A. Haridy (Phd).et.al. Int. Journal of Engineering Research and Application www.ijera.com ISSN : 2248-9622, Vol. 7, Issue 6, (Part -2) June 2017, pp.72-80

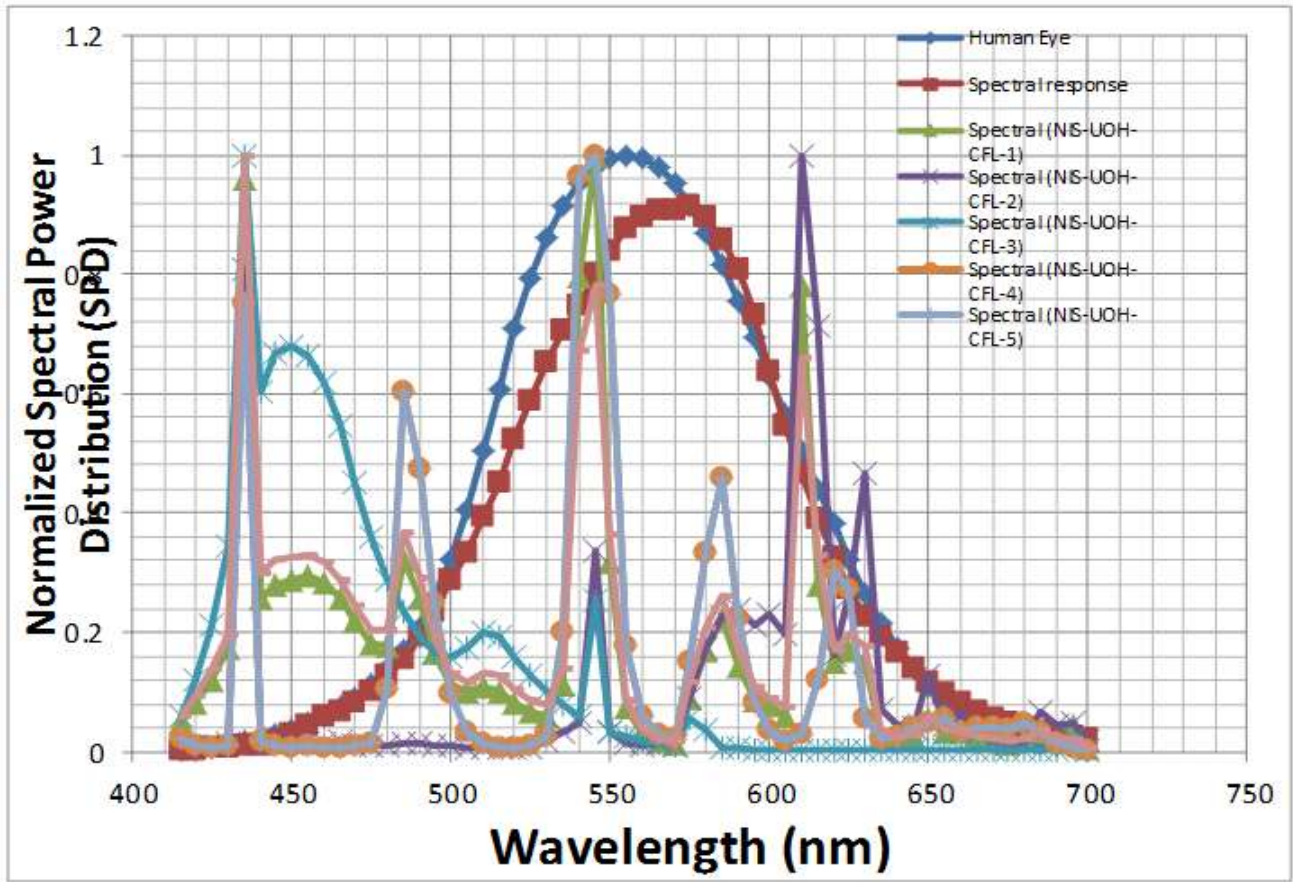

Figure 4. The responsivity of human eye $V(\lambda)$ Curve, spectral photometer response and the spectral power distribution compact lamps (CFLs).

Figure (5) shows spectral power distribution (SPDs) diagrams at each wavelength over the visible region of the NIS-UOH-CFL-1 compact fluorescent. It is used for describing visible spectrum of lighting sources. This quantity shows the radiant power emitted by the source at each wavelength over the visible region (400 to $700 \mathrm{~nm}$ ). The spectral power distribution (SPD) diagram showed typical compact fluorescent lamps (CFLs) response, with a few narrow spectral peaks and it have its own characteristics and it emit its spectrum in the visible region.

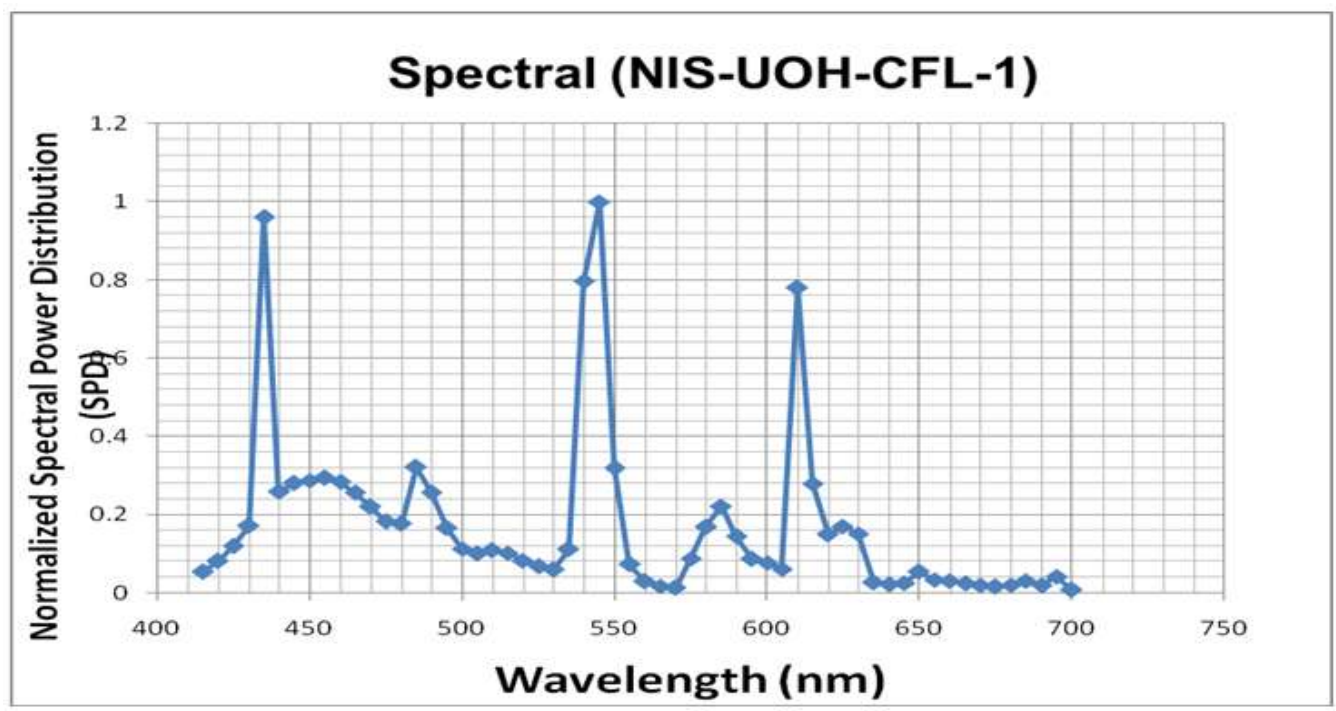

Figure 5. The spectral power distributions of NIS-UOH-CFL-1.

Figure (6) shows the spectral power distribution SPD at each wavelength over the visible region of the NIS-UOH-CFL-2 compact fluorescent. It is used for describing visible spectrum of lighting sources. This quantity shows the radiant power emitted by the source at each wavelength over the visible region (400 to $700 \mathrm{~nm}$ ). The spectral power distribution (SPD) diagram showed typical compact fluorescent lamps (CFLs) response, with a few narrow spectral peaks and it have its own characteristics and it emit its spectrum in the visible region. 


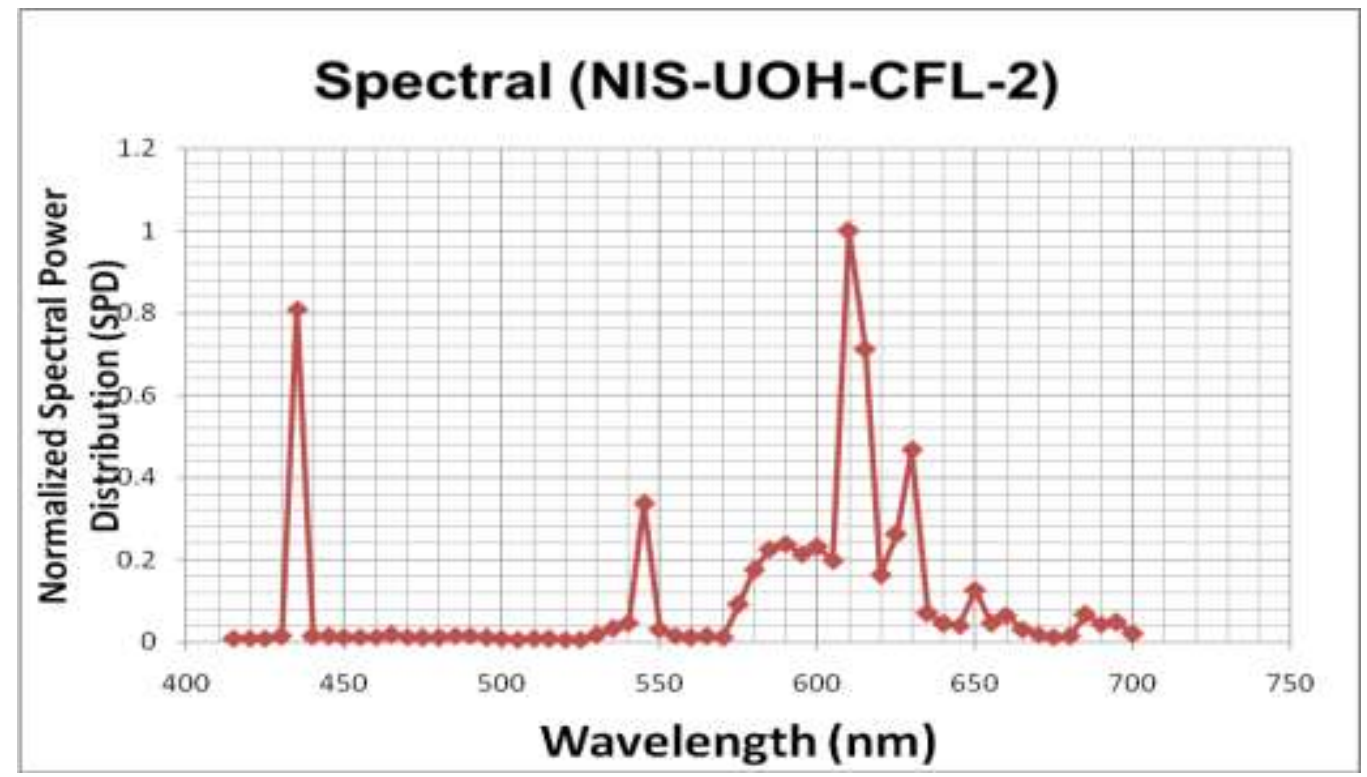

Figure 6. The spectral power distributions of NIS-UOH-CFL-2.

Figure (7) shows the spectral power distribution SPD at each wavelength over the visible region of the NIS-UOH-CFL-3 compact fluorescent. It is used for describing visible spectrum of lighting sources. This quantity shows the radiant power emitted by the source at each wavelength over the visible region (400 to $700 \mathrm{~nm}$ ). The spectral power distribution (SPD) diagram showed typical compact fluorescent lamps (CFLs) response, with a few narrow spectral peaks and it have its own characteristics and it emit its spectrum in the visible region.

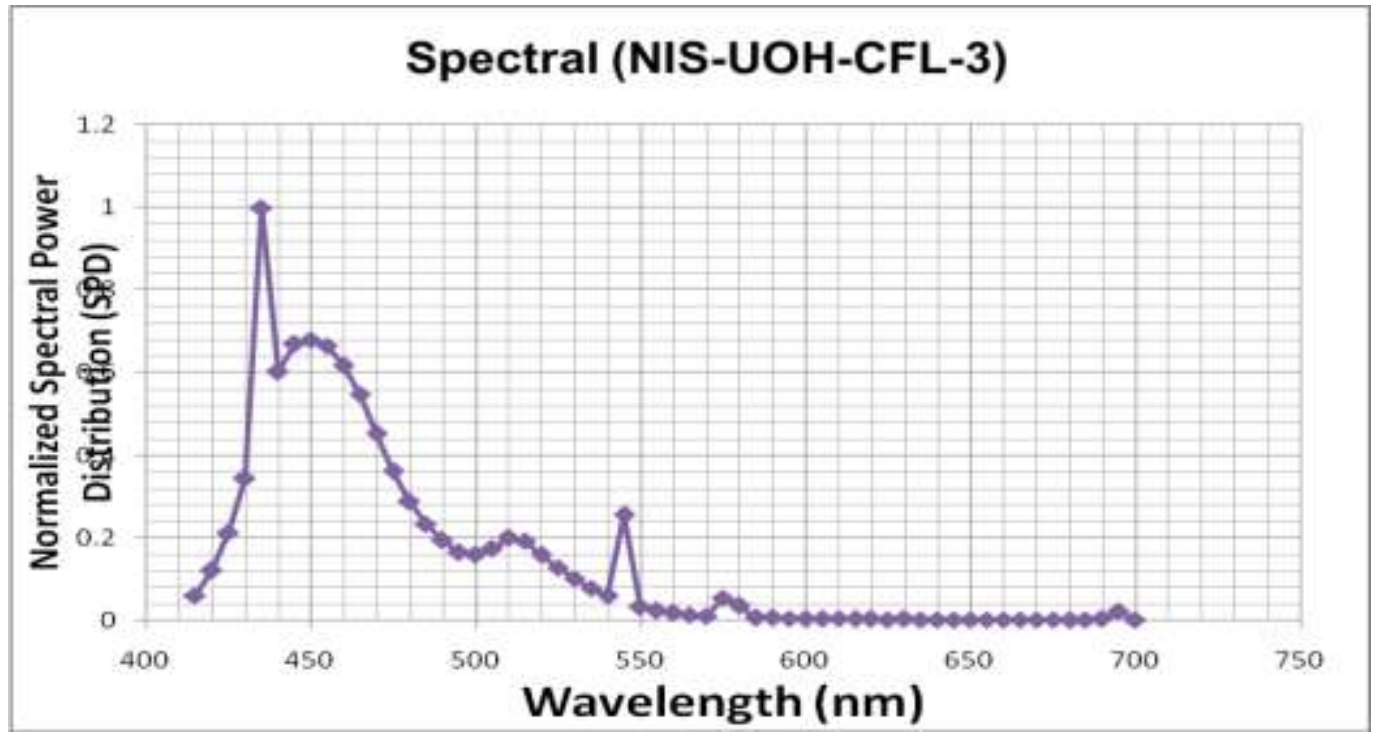

Figure 7. The spectral power distributions of NIS-UOH-CFL-3.

Figure (8) shows the spectral power distribution SPD at each wavelength over the visible region of the NIS-UOH-CFL-4 compact fluorescent. It is used for describing visible spectrum of lighting sources. This quantity shows the radiant power emitted by the source at each wavelength over the visible region (400 to $700 \mathrm{~nm}$ ). The spectral power distribution (SPD) diagram showed typical compact fluorescent lamps (CFLs) response, with a few narrow spectral peaks and it have its own characteristics and it emit its spectrum in the visible region. 


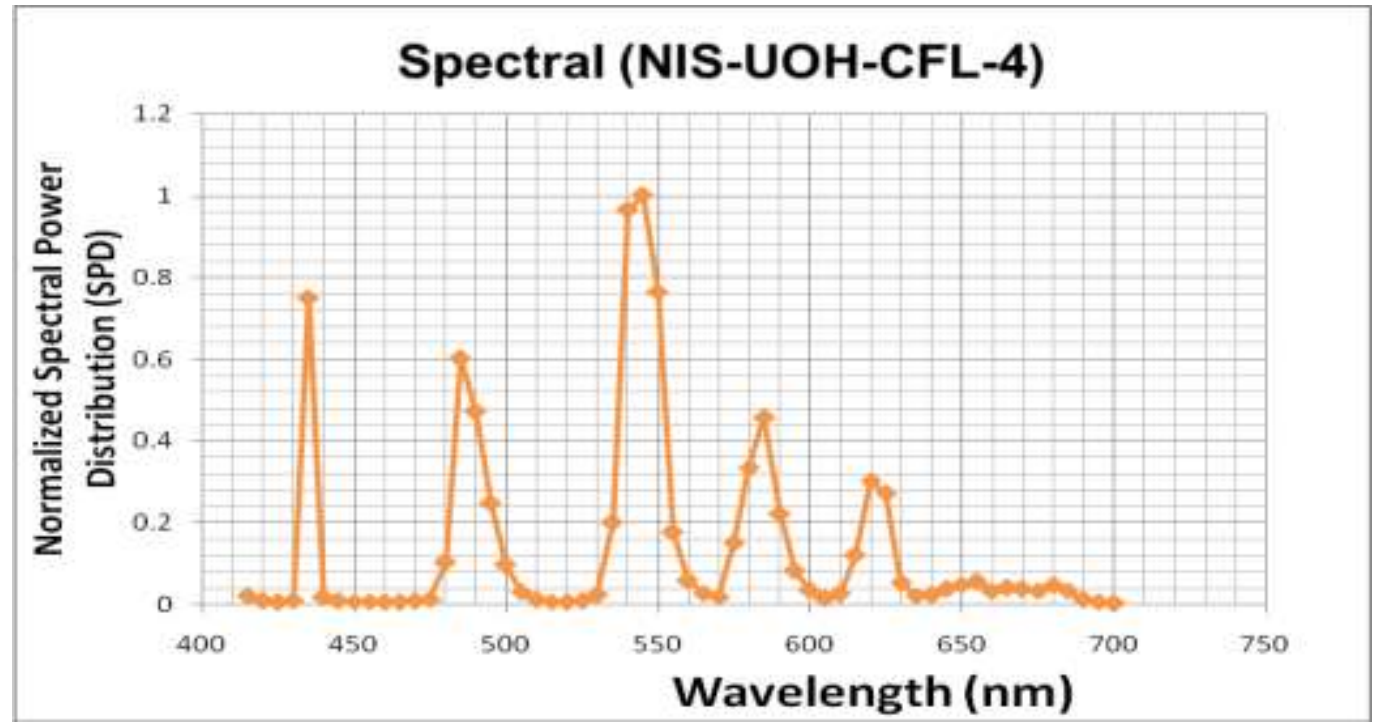

Figure 8. The spectral power distributions of NIS-UOH-CFL-4.

Figure (9) shows the spectral power distribution SPD at each wavelength over the visible region of the NIS-UOH-CFL-5 compact fluorescent. It is used for describing visible spectrum of lighting sources. This quantity shows the radiant power emitted by the source at each wavelength over the visible region (400 to $700 \mathrm{~nm}$ ). The spectral power distribution (SPD) diagram showed typical compact fluorescent lamps (CFLs) response, with a few narrow spectral peaks and it have its own characteristics and it emit its spectrum in the visible region.

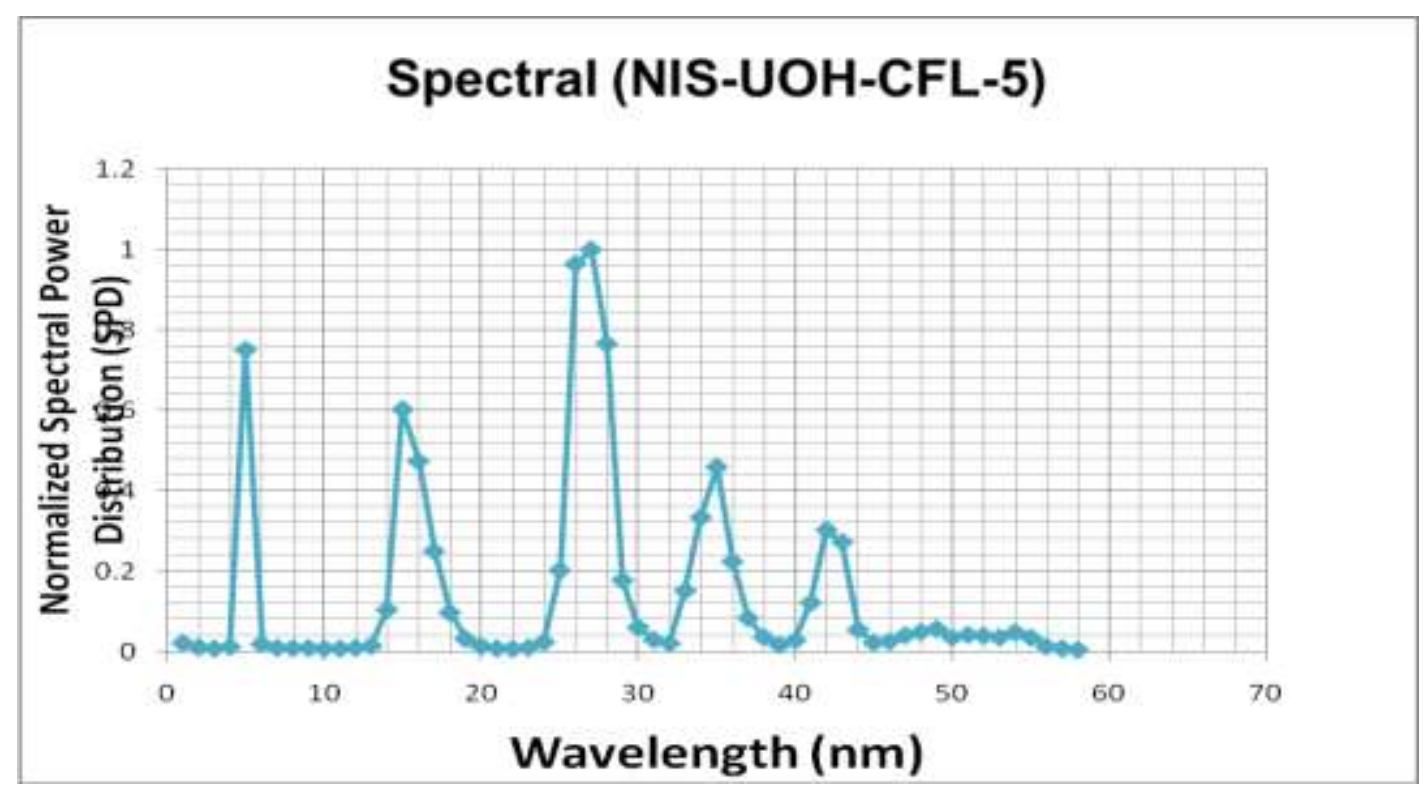

Figure 9. The spectral power distributions of NIS-UOH-CFL-5.

Figure (10) shows the spectral power distribution SPD at each wavelength over the visible region of the NIS-UOH-CFL-6 compact fluorescent. It is used for describing visible spectrum of lighting sources. This quantity shows the radiant power emitted by the source at each wavelength over the visible region (400 to $700 \mathrm{~nm}$ ).
The spectral power distribution (SPD) diagram showed typical compact fluorescent lamps (CFLs) response, with a few narrow spectral peaks and it have its own characteristics and it emit its spectrum in the visible region. 


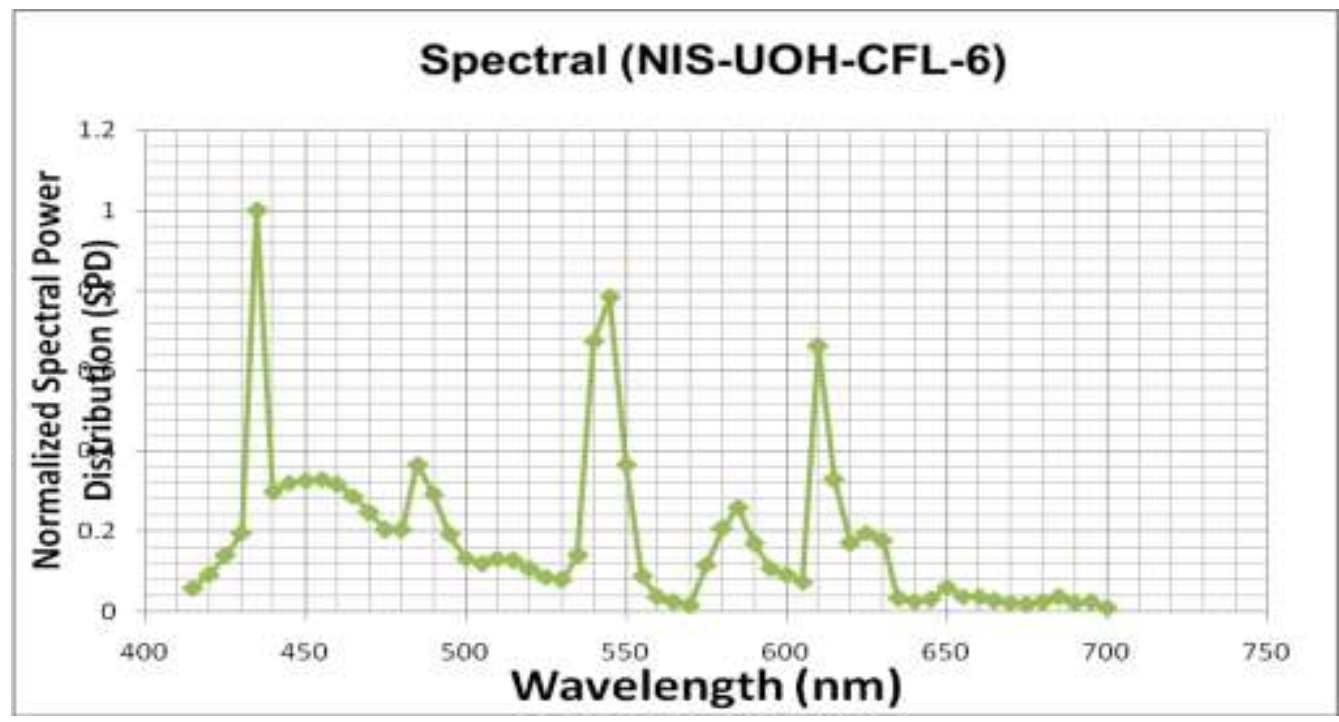

Figure 10. The spectral power distributions of NIS-UOH-CFL-6.

Figure (11). shows the histogram of Spectral Mismatch Factor (SCF) of the six commercial compact fluorescent lamps (CFLs) as the following: NIS-UOH-CFL-1, NIS-UOH-CFL-2, NIS-UOHCFL-3, NIS-UOH-CFL-4, NIS-UOH-CFL-5, NISUOH-CFL-6 depend on their relative spectral power distributions, the spectral power distribution of the human response curve $\mathrm{V}(\lambda)$ and the photometer response. We found that for the lamp NIS-UOH-
CFL-6 the spectral power distribution of the human response curve $\mathrm{V}(\lambda)$ is the lowest value in the other five lamps. According to these results the spectral power distribution of this type of compact lamp does not match perfectly with the spectral power distribution of the human response curve $V(\lambda)$ and the photometer response.

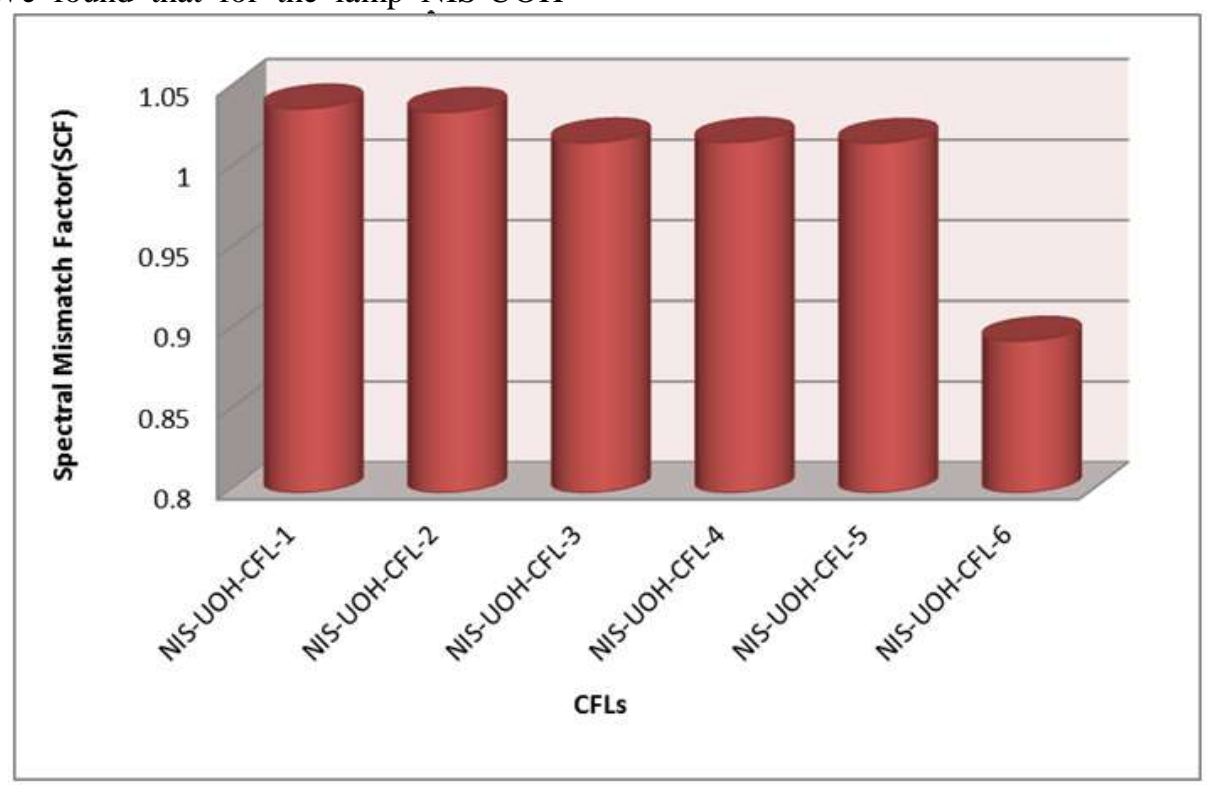

Figure 11. Spectral Mismatch Factor of CFLs.

\section{UNCERTAINTY ANALYSIS}

Modern metrology practice in any field requires accurate estimation of uncertainties. The concept of uncertainty as a quantifiable attribute is relatively new in the history of measurement, although error and error analysis have long been a part of the practice of measurement science or metrology. It is now widely recognized that, when all of the known or suspected components of error have been evaluated and the appropriate corrections have been applied, there still remains an uncertainty about the corrections of the stated result, that is, a doubt about how well the result of the measurement represents the value of the quantity being measured. Consistent estimation of uncertainty according to the methods described in the ISO Guide to the 
Expression of uncertainty in Measurements [13] is a major concern of modern metrology. Evaluation of the uncertainty is done by the Guide to the expression of uncertainty in Measurement (GUM)method. This method is adopted and described in details by

$$
u\left(x_{i}\right)=s(\bar{X})=\left(\frac{1}{n(n-1)} \sum_{k=1}^{n}\left(X_{i, k}-\bar{X}\right)^{2}\right)^{1 / 2}
$$

The combined standard uncertainty $u c(y)$ is obtained by combining the individual standard uncertainties $u_{i}$ these can be evaluated as Type A and Type B. That is,

$$
u_{c}^{2}(y)=\sum_{i=1}^{N}\left(\frac{\partial f}{\partial x_{i}}\right)^{2} u^{2}\left(x_{i}\right)
$$

The accompanied uncertainty of the spectral power distribution (SPDs) measurements for the CFLs lamps is equal to $9.4 \%$ as shown in Table 1 .

Table (1).Estimated Uncertainty of spectral power distribution for the CFLs lamps lamps.

\begin{tabular}{|c|c|}
\hline Uncertainty Component & Relative Standard Uncertainty (\%) \\
\hline Calibration of spectroradiometer & 4.7 \\
\hline Distance measurements & 0.01 \\
\hline Repeatability & 0.02 \\
\hline Relative Expanded Uncertainty $(\boldsymbol{k}=\mathbf{2})$ & $\mathbf{9 . 4 \%}$ \\
\hline
\end{tabular}

\section{CONCLUSIONS}

Lighting comfort is a main factor affecting our ability to perform tasks in closed spaces. Some characteristics of lighting sources can effect on quality of lighting. White light emitting diode lamps can be employed for homes usage and for offices rooms. The spectral power distribution SPDs at each wavelength over the visible region of six of commercial compact fluorescent lamps as the following: NIS-UOH-CFL-1, NIS-UOH-CFL-2, NISUOH-CFL-3, NIS-UOH-CFL-4, NIS-UOH-CFL-5, NIS-UOH-CFL-6 compared to the spectral power distribution of the human response curve $\mathrm{V}(\lambda)$. It is used for describing visible spectrum of lighting sources. This quantity shows the radiant power emitted by the source at each wavelength over the visible region (400 to $700 \mathrm{~nm}$ ). The spectral power distribution (SPDs) diagrams showed typical compact fluorescent lamps (CFLs) response, with a few narrow spectral peaks. It is found that each group of lamps has its own characteristics and they emit their spectrum in the visible region with different spectral distributions for each lamp. The results of Spectral Mismatch Factor (SCF) of the six commercial compact fluorescent lamps (CFLs) depend on their relative spectral power distributions, the spectral power distribution of the human response curve $\mathrm{V}(\lambda)$ and the photometer response. We found that for the lamp NIS-UOH-CFL-6 the spectral power distribution of the human response curve $\mathrm{V}(\lambda)$ is the lowest value in the other five lamps. According to these results the spectral power distribution of this type of compact lamp does not match perfectly with the spectral power distribution of the human response curve $\mathrm{V}(\lambda)$ and the photometer response.

\section{REFERENCES}

[1] Manal A. Haridy, Sameh M. Reda, Abdel Naser Alkamel Mohmed, "Illuminance and Ultra Violet Emissions Radiated from White Compact Fluorescent Lamps", Int. J. Metrol. Qual. Eng. 7, 407 (2016).

[2] C. Figueres, M. Bosi, Achieving greenhouse gas emission reductions in developing countries through energy efficient lighting projects in the clean development mechanism (CDM) (The Carbon Finance Unit, World Bank, Washington, DC, USA, 2006)

[3] I. Abdel Gelil, Regional report on efficient lighting in the Middle East and North Africa, Tech. Rep., Division of Global Environment Facility Coordination, United Nations Environment Programme, Nairobi, Kenya, 2011

[4] P. Waide, S. Tanishima, Light's labour's lost: policies for energy-efficient lighting (OECD Publishing, 2006)

[5] Sullivan, C. R. \& Drescher, A. C. (1993). Influence of on-time and use frequency on cost-effectiveness of compact fluorescent lamps, Conference Record of the 1993 IEEE Industrial Application Society Annual 
Meeting, Toronto, Ontario, Canada, Oct 2-8, 1993, 2283-2290

[6] Azizi M, Golmohammadi R, Aliabadi M. Comparative Analysis of Lighting Characteristics and Ultraviolet Emissions from Commercial Compact Fluorescent and Incandescent Lamps. J Res Health Sci.; 16(4):200-205,2016.

[7] Gloria SC, Sproul AB, Dain SJ. Performance of 'energy efficient' compact fluorescent lamps. Clin Exp Optom; 93(2):66-76., 2010.

[8] CIE 2012. CIE Draft Standard DS 023/E: 2012. Characterization of the Performance of Illuminance Meters and Luminance Meters, Vienna, CIE, 2012.

[9] Manal A. Haridy "Uncertainty estimation of spectral mismatch correction factor for incandescent lamps. Int. J. Curr. Res. Aca. Rev., 3(7): 262 273, 2015.

[10] A.A. Gaertner, "LED Measurement Issues", Photometry, Radiometry And Colorimetry Course NRC, Ottawa, Canada, 2007.

[11] El-Bialy, A.B., El-Ganainy, M.M., ElMoghazy, E.M. 2011. Uncertainty determination of correlated color temperature for high intensity discharge lamps. Nature Sci., 9(3).

[12] International Electrotechnical Commission (IEC). Spectroradiometric measurement of light sources. Vienna: CIE Publication;1984

[13] Guide to the Expression of Uncertainty in Measurement, First Edition, International Organization for Standardization (ISO), 1995.

[14] International Organization for Standardization (ISO), Guide to the expression of uncertainty in measurement (1993).

[15] United Kingdom Accreditation Service (UKAS), The expression of uncertainty and confidence in measurement, 2nd ed. (2007). 